\title{
EFEITO DA APLICAÇÃO SUCESSIVA DE PRECIPITAÇÕES PLUVIAIS COM DIFERENTES PERFIS NA TAXA DE INFILTRAÇÃO DE ÁGUA NO SOLO(1)
}

\author{
João Henrique Zonta ${ }^{(2)}$, Mauro Aparecido Martinez ${ }^{(3)}$, Fernando Falco \\ Pruski $^{(3)}$, Demetrius David da Silva ${ }^{(3)} \&$ Marcelo Rocha dos Santos ${ }^{(4)}$
}

\section{RESUMO}

O processo de infiltração é influenciado pelas condições da superfície do solo e pela precipitação pluvial. O objetivo deste trabalho foi avaliar a influência de aplicações sucessivas de precipitações pluviais com diferentes perfis na formação do encrostamento superficial e, consequentemente, na taxa de infiltração de água em solo sem cobertura e com cobertura. Foram aplicadas três precipitações pluviais sucessivas para cada perfil de precipitação pluvial, em intervalos de $24 \mathrm{~h}$, sendo usados os perfis de precipitação pluvial constante, exponencial decrescente, duplo exponencial adiantado e atrasado, com uma lâmina média de $55 \mathrm{~mm}$ por aplicação. Buscou-se ajustar um fator de decaimento da taxa de infiltração (Ti) em função da energia cinética acumulada da chuva, denominado de fator $f$, dado pela razão entre a taxa de infiltração estável (Tie) com efeito da chuva e a Tie sem o efeito da chuva. Foram avaliadas duas condições de cobertura do solo, solo sem cobertura e solo coberto com palhada, sendo os ensaios de infiltração realizados com um simulador de chuvas, em parcelas experimentais de dimensões de $1,0 \times 0,7 \mathrm{~m}$. Os resultados foram avaliados por meio de análises gráficas, análise de variância e teste de média. Verificou-se que os diferentes perfis de precipitação pluvial não influenciaram a infiltração de água no solo, tanto para o solo sem cobertura como para o solo com cobertura, sendo esta influenciada somente pelas aplicações sucessivas, com menores valores da taxa de infiltração obtidos na segunda e terceira aplicações. O decréscimo na Ti em

\footnotetext{
(1) Parte de Tese de Doutorado apresentada ao DEA/UFV. Recebido para publicação em 15 de fevereiro de 2011 e aprovado em 9 de janeiro de 2012.

(2) Pesquisador da Embrapa Algodão. Rua Oswaldo Cruz 1143, Centenário, Caixa Postal 174, CEP 58428-095 Campina Grande (PB). E-mail: zonta@cnpa.embrapa.br

(3) Professor do DEA/UFV, Campus Universitário s/n, Centro, CEP 36570-000 Viçosa (MG). E-mails: mmauro@ufv.br; ffpruski@ufv. br; demetrius@ufv.br

(4) Professor do Instituto Federal de Educação, Ciência e Tecnologia Baiano - IFBAIANO. Rua do Rouxinol 115, Imbuí, CEP 41720052 Salvador (BA). E-mail: marrochas@yahoo.com.br
} 
solo sem cobertura foi devido à formação de encrostamento superficial, o que ocorreu logo na primeira aplicação. A Tie em solo sem cobertura teve decréscimo de 75 \% se comparada à Tie em solo com cobertura. A lâmina infiltrada não foi influenciado pelos quatro perfis de precipitação pluvial. O fator $f$ foi descrito com uso de uma equação do tipo exponencial.

Termos de indexação: cobertura do solo, encrostamento superficial, energia cinética da chuva.

\title{
SUMMARY: EFFECT OF SUCCESSIVE RAINFALL WITH DIFFERENT PATTERNS ON SOIL WATER INFILTRATION RATE
}

\begin{abstract}
The purpose of this study was to evaluate the influence of successively repeated precipitation patterns applied to bare and to covered soil, to quantify their effect on the formation of a soil crust and, consequently, on the soil water infiltration rate (Ti). Three rainfall events were simulated in $24 \mathrm{~h}$ intervals, with constant, decreasing exponential, early and late double exponential precipitation patterns, at a rainwater amount corresponding to $55 \mathrm{~mm}$. The decrease factor of the infiltration rate (Ti) was adjusted as a function of the accumulated kinetic energy of the rainwater, called factor $f$, given by the ratio between the stable infiltration rate (Tie) affected by rain, and Tie with no effect of rain. Two situations of soil cover were evaluated: bare soil and soil covered with crop residues. A rainfall simulator was used in the infiltration experiments, on $1.0 \times 0.7 \mathrm{~m}$ plots. The results were evaluated by graphical analysis, analysis of variance and means testing. It was found that the different precipitation profiles did not affect Ti of bare or covered soil. The Ti values were only influenced by successive rainfall, and the water infiltration rate was lowest after the $2^{\text {nd }}$ and $3^{\text {rd }}$ application. The decrease in Ti of bare soil was due to a crust on the soil surface, which was first formed after the $1^{\text {st }}$ application. The stable infiltration rate (Tie) on bare soil decreased by $75 \%$ when compared to Tie of covered soil. The infiltration depth was not influenced by any of the tested rainfall patterns. The factor $f$ was described by an exponential equation.
\end{abstract}

Index terms: soil cover; soil crusting; kinetic energy of rainfall.

\section{INTRODUÇÃO}

O processo de infiltração de água no solo é um importante componente do ciclo hidrológico, pois, junto com a precipitação pluvial, determina a quantidade de água que se torna disponível para as plantas, o escoamento superficial e o abastecimento dos reservatórios de águas subterrâneas. É um processo que tende a um valor constante, denominado de taxa de infiltração estável (Tie) ou taxa de infiltração básica (Araújo Filho \& Ribeiro, 1996) - atributo esse que é de fundamental importância para definir os métodos de conservação do solo, planejamento e dimensionamento de sistemas de irrigação e drenagem (Cunha, 2009).

Segundo Pott \& De Maria (2003), vários fatores condicionam a infiltração de água no solo, como porosidade, densidade do solo, cobertura do solo, textura do solo, encrostamento superficial, umidade inicial, matéria orgânica, estrutura e variabilidade espacial do terreno. É um processo físico complexo, sobretudo nas condições normalmente encontradas de anisotropia e heterogeneidade dos solos.

O encrostamento superficial, causado pelo impacto das gotas de água, é um processo de compactação superficial resultante do impacto das gotas de chuva em solos intensivamente cultivados. Ele é resultante de processos complexos e dinâmicos, nos quais as partículas do solo são rearranjadas e consolidadas em uma estrutura coesa, cuja espessura pode variar de 0,1 a $50 \mathrm{~mm}$, e seu efeito sobre as propriedades físicas do solo, principalmente sobre a condutividade hidráulica, influencia, acentuadamente, as condições de infiltração.

Le Bissonnais et al. (2005) afirmam que, em solos sem cobertura vegetal ou cultivados com aração e gradagem, o encrostamento superficial tem influência muito forte nas propriedades hidráulicas do solo, como a taxa de infiltração, que pode ser consideravelmente reduzida; a magnitude dessa redução depende das características do solo, como textura, estrutura, teor de matéria orgânica 
e estabilidade dos agregados, e das características da chuva, como intensidade e duração (Morin et al., 1989). Brandão et al. (2006a) mencionam reduções de até $85 \%$ na taxa de infiltração em função da formação de encrostamento superficial.

Para Truman et al. (2007), ocorrem maiores mudanças na superfície do solo quando perfis de precipitação pluvial com intensidade variável são aplicados, se comparados ao perfil de precipitação pluvial constante. Flanagan et al. (1987) afirmam que uma alta intensidade de precipitação pluvial no início do evento pode causar maior compactação e formação de encrostamento da superfície do solo. Esse comportamento deve-se à quebra dos agregados tanto pela força mecânica do impacto das gotas de chuva como pelo rápido umedecimento e rachadura dos agregados, devido à alta intensidade de precipitação pluvial inicial e ao rápido umedecimento.

De Roo \& Riezebos (1992) e Robinson \& Woodun (2008) avaliaram o efeito de duas chuvas sucessivas com intervalo de $24 \mathrm{~h}$ e afirmaram que ocorreu incremento na densidade do solo na superfície e formação de encrostamento superficial já na primeira precipitação pluvial, e que isso resultou em decréscimo expressivo na taxa de infiltração na precipitação pluvial subsequente.

Alguns autores buscaram descrever os efeitos dessas precipitações pluviais sucessivas na taxa de infiltração de água no solo. Risse et al. (1995) e Silva \& Kato (1997) observaram que o decréscimo dos valores da condutividade hidráulica do solo com as precipitações pluviais consecutivas relacionam-se bem aos valores da energia cinética acumulada da chuva. Dessa forma, torna-se importante a análise do efeito de precipitações pluviais sucessivas, aplicadas com diferentes perfis, na formação da camada de crosta e no processo de infiltração.

Diante do exposto, o presente trabalho teve como objetivo estudar o efeito de sucessivas aplicações de chuvas simuladas com diferentes perfis de precipitação pluvial na taxa de infiltração de água no solo, estando a superfície com e sem cobertura vegetal.

\section{MATERIAL E MÉTODOS}

Os ensaios de infiltração foram realizados na área experimental do Departamento de Engenharia Agrícola da Universidade Federal de Viçosa - MG. O solo da área foi classificado como Cambissolo Háplico Tb distrófico, com declividade média de 8,0 \%, com horizonte A de textura argiloarenosa (55\% de areia, $39 \%$ de argila e $6 \%$ de silte) e horizonte B de textura argilosa ( $40 \%$ de areia, $53 \%$ de argila e $7 \%$ de silte) (Quadro 1). Previamente à instalação das parcelas experimentais, foi realizado o preparo convencional do solo, com aração e gradagem.
Quadro 1. Massa específica do solo ( $\rho)$, massa específica das partículas $\left(\rho_{\mathrm{r}}\right)$, porosidade total $(\varepsilon)$ e condutividade hidráulica do solo saturado $\left(K_{0}\right)$ do perfil do solo

\begin{tabular}{lcccc}
\hline Horizonte & $\boldsymbol{\rho}^{(1)}$ & $\boldsymbol{\rho}_{\mathbf{r}}^{(2)}$ & $\boldsymbol{\varepsilon}\left(\boldsymbol{\theta}_{\mathrm{S}}\right)^{(3)}$ & $\mathbf{K}_{\mathbf{0}}{ }^{(4)}$ \\
\hline & \multicolumn{2}{c}{$\mathrm{kg} \mathrm{dm}^{-3}$} & $\mathrm{dm}^{3} \mathrm{dm}^{-3}$ & $\mathrm{~mm} \mathrm{~h}^{-1}$ \\
A (0-30 cm) & 1,40 & 2,57 & 0,46 & 40,0 \\
B (abaixo de 30 cm) & 1,37 & 2,58 & 0,47 & 20,0 \\
\hline
\end{tabular}

(1) Método do anel volumétrico. ${ }^{(2)}$ Método do balão volumétrico. (3) Umidade do solo saturado. ${ }^{(4)}$ Método do permeâmetro de carga constante.

Os simuladores de chuvas utilizados (Figura 1a), desenvolvidos no DEA/UFV, eram similares ao descrito por Meyer \& Harmon (1979), tendo cinco bicos VeeJet 80.100 oscilantes e dispostos linearmente, instalados a uma altura de $3 \mathrm{~m}$ da superfície do solo, trabalhando com pressão de serviço de $41,4 \mathrm{kPa}$. Esse equipamento possui a capacidade de aplicar precipitações pluviais com intensidades variadas ao longo do tempo, o que possibilita a aplicação de precipitações pluviais com diferentes perfis, sendo a intensidade controlada por um circuito analógico-digital conectado a um microcomputador. A calibração do simulador de chuvas foi realizada seguindo método apresentado por Zonta et al. (2007).

Foram dispostos na área experimental dois simuladores de chuvas e, sob cada um deles, instaladas quatro parcelas experimentais, delimitadas com chapas metálicas com dimensões de $0,7 \mathrm{~m}$ de largura por 1,0 $\mathrm{m}$ de comprimento e $0,18 \mathrm{~m}$ de altura, enterradas $0,10 \mathrm{~m}$ no solo, distanciadas de $0,3 \mathrm{~m}$ uma da outra (Figura 1b).

O escoamento superficial produzido durante o ensaio foi conduzido - por meio de uma calha conectada a um tubo flexível de $50 \mathrm{~mm}$ de diâmetro - para um recipiente de coleta, no qual foi instalado um registrador de nível de água, denominado Thalimedes, que registrava a altura da lâmina de água dentro do recipiente. A taxa de infiltração a cada minuto foi determinada pela diferença entre a intensidade de precipitação pluvial aplicada e a taxa de escoamento superficial gerado.

Os ensaios de infiltração consistiram na aplicação de chuva simulada, seguindo quatro perfis de distribuição da intensidade de precipitação pluvial ao longo do tempo: constante (caracterizado por uma intensidade de precipitação pluvial constante ao longo do tempo), exponencial decrescente (caracterizado por uma intensidade de precipitação pluvial que decresce ao longo da duração), duplo exponencial adiantado (caracterizado pela ocorrência da intensidade máxima de precipitação pluvial no tempo correspondente a $30 \%$ da duração do 
(a)

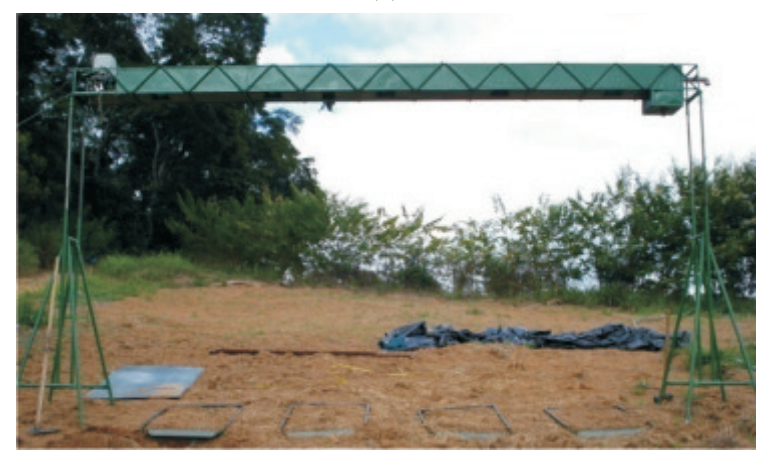

(b)

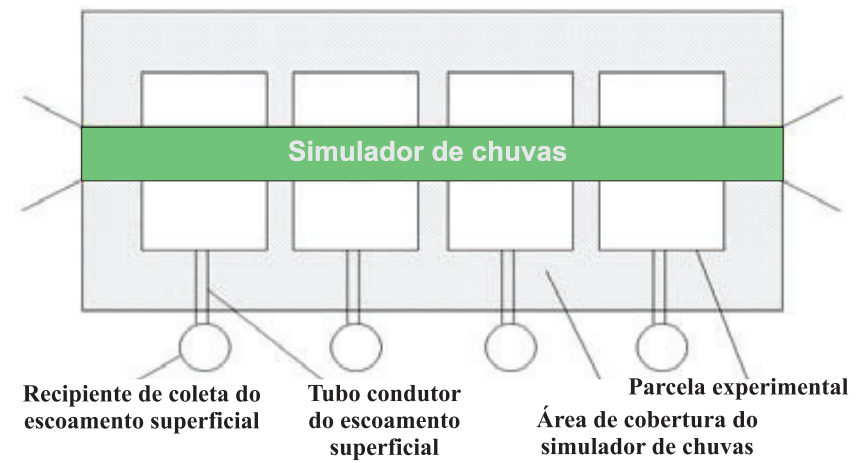

Figura 1. Simulador de chuvas utilizado no experimento (a) e arranjo das parcelas para a realização dos testes de infiltração (b).

evento) e atrasado (caracterizado pela ocorrência da intensidade máxima de precipitação pluvial no tempo correspondente a $70 \%$ da duração do evento) (Figura 2), em solo sem cobertura e com cobertura vegetal. As equações que descrevem os perfis de precipitação pluvial utilizados neste trabalho podem ser encontradas em Montebeller (2009). A cobertura do solo foi feita com uso de palha seca formada por capim Coast-Cross, na quantidade de $5 \mathrm{t} \mathrm{ha}^{-1}$, equivalente a cobertura recomendada de aproximadamente $80 \%$ da superfície no sistema plantio direto (Embrapa, 2006).

Os perfis de precipitação pluvial foram ajustados de modo que fosse aplicada uma lâmina total de $55 \mathrm{~mm}$, tendo os ensaios duração de $30 \mathrm{~min}$, com intensidade máxima média de precipitação pluvial determinada com uso da equação de intensidadeduração e frequência, para a região de Viçosa-MG, para um período de retorno de 15 anos, que é atualmente o mais indicado para dimensionamento de estruturas de conservação de solo e água, como terraceamento. Foram aplicadas para cada perfil de precipitação pluvial três chuvas sucessivas com intervalos de $24 \mathrm{~h}$, sendo nesse intervalo o solo mantido coberto, a fim de evitar a evaporação.

A energia cinética acumulada da chuva para cada um dos perfis de precipitação pluvial aplicados foi

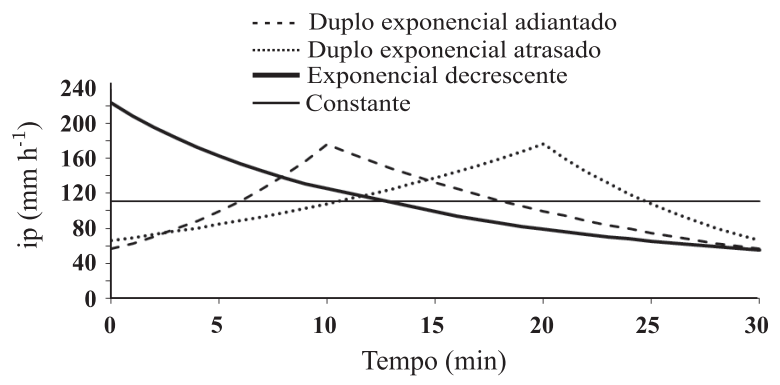

Figura 2. Perfis de precipitação pluvial utilizados no experimento. calculada conforme método proposto por Wischmeier \& Smith (1958):

$$
\mathrm{Ec}=0,119+0,0873 \log (\mathrm{I})
$$

em que Ec é a energia cinética das gotas de chuvas $\left(\mathrm{MJ} \mathrm{ha}^{-1} \mathrm{~mm}^{-1}\right.$ ) e I é a intensidade de precipitação pluvial $\left(\mathrm{mm} \mathrm{h}^{-1}\right)$.

A equação 1 foi aplicada a cada intervalo de $1 \mathrm{~min}$, no qual a intensidade de precipitação pluvial era constante, sendo o resultado obtido multiplicado pela lâmina precipitada em cada intervalo; em seguida, foi realizada a soma de todos os valores para cada perfil de precipitação pluvial, obtendo-se com isso a energia cinética acumulada para cada perfil de precipitação pluvial. Nas aplicações sucessivas foi considerado que inicialmente já havia sido aplicada a energia cinética acumulada da aplicação anterior; assim, no final da terceira aplicação, tem-se como energia cinética acumulada o total das três aplicações.

Houve algumas variações na lâmina total aplicada em função de limitações do simulador de chuvas e efeitos de ventos, porém, pela análise de variância, os valores da energia cinética da chuva acumulada não diferiram, a $5 \%$, entre os quatro perfis de precipitação pluvial, sendo aplicados em média 154,315 e $460 \mathrm{~J} \mathrm{~m}^{-2}$, respectivamente, para a primeira, segunda e terceira aplicações.

Em cada parcela experimental foram instaladas cinco sondas TDR (reflectometria no domínio do tempo) nas profundidades de 10, 20, 30, 40 e $50 \mathrm{~cm}$, para a medição do conteúdo de água a cada minuto no solo durante os ensaios de infiltração. Para isso, foi montado um conjunto com quatro multiplexadores SDMX 50SP, conectados a uma TDR 100, e esta conectada a um Datalogger CR10X, todos da Campbell Scientific. As sondas TDR foram calibradas seguindo o método proposto por Santos et al. (2010). Os valores médios do conteúdo de água no solo no início dos ensaios de infiltração foram de 0,27, 0,32 e 0,33 $\mathrm{m}^{3} \mathrm{~m}^{-3}$, respectivamente, para a primeira, segunda e terceira aplicações. 
$\mathrm{O}$ experimento foi instalado num esquema em parcelas subdivididas, tendo os perfis de precipitação pluvial como parcelas e as aplicações sucessivas como subparcelas. O delineamento experimental foi em blocos casualizados (DBC), com três repetições, totalizando 36 ensaios de infiltração. Cada perfil de precipitação pluvial foi aplicado em uma das parcelas instaladas sob o simulador de chuvas, sendo os perfis de precipitação pluvial distribuídos nas parcelas ao acaso. Foi realizado um experimento para o solo sem cobertura e outro para o solo com cobertura. Os resultados foram avaliados por meio de análises gráficas, análise de variância e teste de Tukey a $5 \%$.

Para o solo com cobertura, foi realizado um ensaio de infiltração com intensidade constante de $160 \mathrm{~mm} \mathrm{~h}^{-1}$, até que fosse alcançada a taxa de infiltração estável, sendo o valor da Tie determinado igual a $60 \mathrm{~mm} \mathrm{~h}^{-1}$. Para quantificar o efeito da energia cinética acumulada da chuva (Ec) no encrostamento superficial e na taxa de infiltração de água no solo, foi determinado um fator de decaimento da taxa de infiltração estável (Tie) em função da energia cinética acumulada da chuva (Ec), denominado de fator $f$. Esse fator foi determinado porque, dessa forma, em modelos de simulação hidrológica, pode-se trabalhar com o valor da Tie variando no tempo, e não com um valor constante, conforme considerado na maioria dos modelos, o que pode causar superestimativa da lâmina infiltrada; assim, torna-se importante o uso de algum fator de correção da Tie ao longo do tempo, principalmente em condições de solo descoberto.

Para obtenção do fator $f$ foi utilizado valor da Tie obtida em solo com cobertura $\left(60 \mathrm{~mm} \mathrm{~h}^{-1}\right)$, ou seja, sem impacto das gotas de chuva, e a Tie obtida na segunda e terceira aplicações de precipitação pluvial, ou seja, para vários valores de energia cinética acumulada da chuva aplicada na superfície do solo sem cobertura, com o impacto direto das gotas de chuva. O índice $f$ para cada valor de energia cinética acumulada da chuva (Ec) foi calculado pela seguinte equação:

$$
f=\frac{T i e(E c)}{T i e_{\text {cobertura }}}
$$

em que Tie $(\mathrm{Ec})$ = taxa de infiltração estável obtida para um determinado valor de energia cinética acumulada da chuva, em solo sem cobertura, $\mathrm{mm} \mathrm{h}^{-1}$; e Tie $e_{\text {cobertura }}=$ taxa de infiltração estável obtida em solo com cobertura, $\mathrm{mm} \mathrm{h}^{-1}$.

Com os pares de dados de Ec e $f$ (obtidos para a segunda e terceira aplicações de cada perfil de precipitação), foi gerada uma equação do tipo exponencial (3), que fornece o valor de $f$ em função da energia cinética acumulada da chuva (Ec). A respectiva equação é da forma:

$$
f=\alpha e^{-\beta E c}+\mathrm{C}
$$

em que $\alpha, \beta$ e C são parâmetros da equação.

\section{RESULTADOS E DISCUSSÃO}

Na figura 3 são apresentadas as curvas da taxa de infiltração média de água no solo para os três ensaios de infiltração sucessivos, aplicados em intervalos de $24 \mathrm{~h}$, em solo com cobertura, para os quatro perfis de precipitação pluvial.

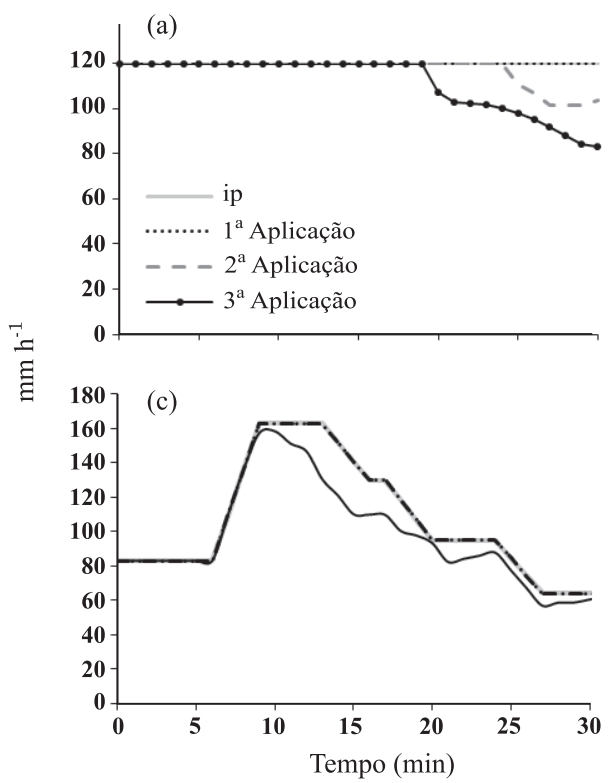

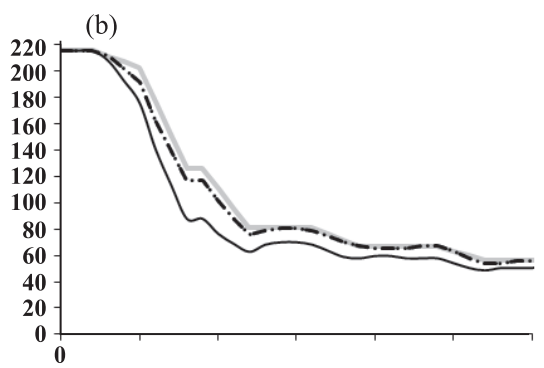

(d)

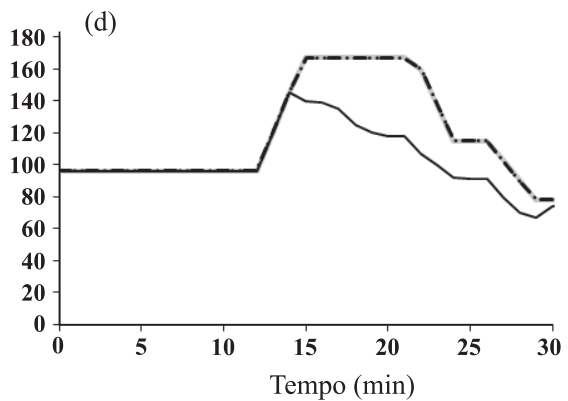

Figura 3. Taxa de infiltração (Ti) para os três ensaios experimentais realizados consecutivamente em solo com cobertura, espaçados $24 \mathrm{~h}$ entre si, com perfil de precipitação pluvial constante (a), exponencial decrescente (b), duplo exponencial adiantado (c) e duplo exponencial atrasado (d). 
A taxa de infiltração de água no solo para os ensaios realizados em solo com cobertura vegetal na superfície apresentou uma mesma tendência para os quatro perfis de precipitação pluvial avaliados, com praticamente toda lâmina aplicada infiltrandose no solo na primeira aplicação, e ocorrência de escoamento superficial, em pequena magnitude, na segunda e terceira aplicações, sendo mais intenso nesta última.

Para os perfis de precipitação pluvial com intensidade variada ao longo do tempo, a formação de escoamento superficial mais acentuado ocorreu nos momentos do pico de intensidade de precipitação pluvial (ip) ou intensidades acima de $100 \mathrm{~mm} \mathrm{~h}^{-1}$; por outro lado, com a diminuição da intensidade de precipitação pluvial, o escoamento superficial cessou ou diminuiu consideravelmente. Para o perfil constante, o escoamento teve início no final dos ensaios, porém a taxa de infiltração estável não foi alcançada.

O fato de o escoamento superficial ter ocorrido na segunda ou terceira aplicações e não na primeira deuse em razão de o solo apresentar conteúdo de água inicial mais elevado nessas aplicações em relação à primeira, possuindo assim menor capacidade de infiltração, devido ao menor gradiente hidráulico e também à menor capacidade de armazenamento de água pelo solo.

Pode-se explicar a relação de causa e efeito do escoamento superficial no final dos ensaios realizados com perfis de precipitação pluvial com intensidade variada ao longo do tempo ter cessado ou diminuído muito sua intensidade, visto que o valor da intensidade de precipitação pluvial nesse instante variava de 50 a $60 \mathrm{~mm} \mathrm{~h}^{-1}$, valor muito próximo à Tie, que era de $60 \mathrm{~mm} \mathrm{~h}^{-1}$. Simões et al. (2005), em estudos em um Cambissolo, encontraram valores da taxa de infiltração estável, com uso de simulador de chuvas, de $57,7 \mathrm{~mm} \mathrm{~h}^{-1}$ - valores próximos aos encontrados neste trabalho.

A mesma tendência apresentada entre as taxas de infiltração, com diferentes perfis de precipitação pluvial, sugere que, em condições de superfície do solo bem protegida contra o impacto direto das gotas de chuva, o perfil de precipitação pluvial não influencia a taxa de infiltração, sendo ele influenciado somente pelas precipitações pluviais sucessivas, ou seja, pela lâmina total aplicada. Esse resultado pode ser comprovado pela não significância na análise estatística pelo teste de Tukey para os perfis de precipitação pluvial (Quadro 2).

Os valores da taxa de infiltração média no tempo nos três ensaios de infiltração sucessivos, aplicados em intervalos de $24 \mathrm{~h}$, em solo sem cobertura, nos quatro perfis de precipitação pluvial, são apresentados na figura 4 . Observa-se (Figura 4a,d) uma característica comum nos quatro perfis de precipitação pluvial: ocorrência de escoamento superficial na metade final do ensaio de infiltração na primeira aplicação e tendência de uma queda abrupta nos valores de taxa de infiltração com as aplicações sucessivas, com rápida formação de escoamento superficial.

Essa característica pode ser explicada pela elevação do conteúdo de água no solo antes da realização dos ensaios, que é maior na segunda e terceira aplicações do que na primeira, acarretando diminuição do gradiente hidráulico e da capacidade de armazenamento de água no solo e, como consequência, da capacidade de infiltração, bem como pela formação de encrostamento superficial, devido à quebra dos agregados pelo impacto direto das gotas de chuva, que pode causar substancial diminuição da condutividade hidráulica da camada superficial do solo, diminuindo bruscamente a taxa de infiltração.

Segundo Pruski et al. (1997), a taxa de infiltração diminui com o número de aplicações de água ao solo e com o aumento da precipitação pluvial total aplicada, independentemente da intensidade da precipitação pluvial. Magunda et al. (1997) aplicaram três chuvas consecutivas com intensidade de precipitação pluvial de $63 \mathrm{~mm} \mathrm{~h}^{-1} \mathrm{em}$ amostras de solos de várias texturas e observaram que, no

Quadro 2. Valores médios da relação entre a lâmina infiltrada e a lâmina aplicada (I/L) para as três aplicações dos diferentes perfis de precipitação pluvial em solo com cobertura

\begin{tabular}{|c|c|c|c|}
\hline \multirow{2}{*}{$\begin{array}{l}\text { Perfil de precipitação } \\
\text { pluvial }\end{array}$} & \multicolumn{3}{|c|}{ Relação entre a lâmina infiltrada (I) e a lâmina aplicada (L) } \\
\hline & $1^{\mathrm{a}}$ aplicação & $2^{a}$ aplicação & $3^{a}$ aplicação \\
\hline Constante & $0,92 \mathrm{a}$ & $0,79 \mathrm{a}$ & $0,72 \mathrm{~b}$ \\
\hline Exponencial & $0,96 \mathrm{a}$ & $0,84 \mathrm{a}$ & $0,69 \mathrm{~b}$ \\
\hline Adiantado & $1,00 \mathrm{a}$ & $1,00 \mathrm{a}$ & $0,85 \mathrm{~b}$ \\
\hline Atrasado & $1,00 \mathrm{a}$ & $1,00 \mathrm{a}$ & $0,80 \mathrm{~b}$ \\
\hline Média & $0,97 \mathrm{~A}$ & $0,91 \mathrm{~A}$ & $0,76 \mathrm{~B}$ \\
\hline
\end{tabular}

Médias seguidas da mesma letra minúscula nas colunas e maiúsculas nas linhas não diferem entre si ao nível de $5 \%$ pelo teste de Tukey. 

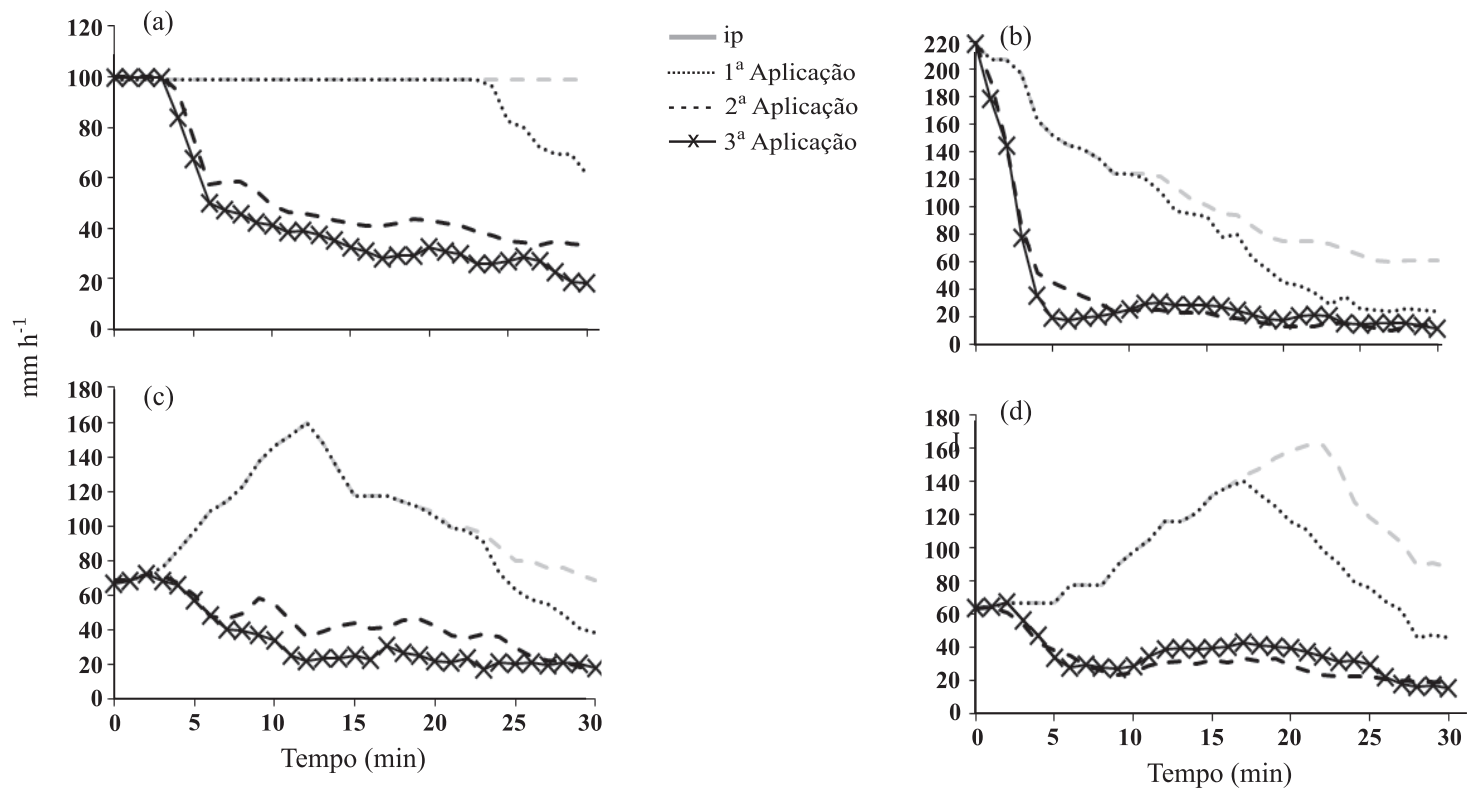

Figura 4. Taxa de infiltração (Ti) para os três ensaios experimentais realizados consecutivamente em solo sem cobertura, espaçados de $24 \mathrm{~h}$ entre si, com perfil de precipitação pluvial constante (a), exponencial decrescente (b), duplo exponencial adiantado (c) e duplo exponencial atrasado (d).

final dos testes, as chuvas simuladas consecutivas causaram decréscimo na taxa de infiltração de água no solo. Para um solo de textura idêntica ao deste estudo, os autores encontraram redução na Tie de $60 \mathrm{~mm} \mathrm{~h}^{-1}$ na primeira precipitação pluvial para $15 \mathrm{~mm} \mathrm{~h}^{-1}$ no final da terceira precipitação pluvial - resultados próximos aos encontrados neste estudo. Outros autores, como Silva (2007), observaram o mesmo comportamento, concluindo que essa redução na taxa de infiltração com as precipitações pluviais sucessivas foi devida não somente ao maior conteúdo de água no solo, mas também ao efeito do encrostamento superficial formado.

Observou-se ainda que as taxas de infiltração na segunda e terceira aplicações foram similares para um mesmo perfil de precipitação pluvial, levando a deduzir que o encrostamento superficial foi formado já com a primeira aplicação, exercendo papel fundamental no comportamento da infiltração de água no solo nos ensaios subsequentes. Robinson $\&$ Woodun (2008) avaliaram o efeito de duas chuvas sucessivas no intervalo de $24 \mathrm{~h}$ e afirmaram que houve incremento na densidade do solo na superfície e formação de encrostamento superficial na primeira aplicação e que isso resultou em decréscimo significativo na taxa de infiltração na aplicação de precipitação pluvial subsequente (de 72,49 para $58,95 \%$ ), a qual foi realizada com mesma intensidade e duração. De Roo \& Riezebos (1992) afirmam que, quando se aplicam precipitações pluviais sucessivas ao solo, a maior porção da camada de encrostamento se desenvolve logo com a aplicação inicial; o efeito das chuvas subsequentes é menos relevante no decréscimo da taxa de infiltração, o que corrobora os resultados encontrados neste experimento.

Outro fator relevante é o fato de que, no final dos ensaios, a taxa de infiltração tendeu a apresentar valor muito próximo na segunda e terceira aplicações, para os quatro perfis de precipitação pluvial - valor esse que variou de 10 a $30 \mathrm{~mm} \mathrm{~h}^{-1}$. Deve-se lembrar que essas pequenas diferenças são esperadas, visto que cada ensaio foi realizado em uma parcela diferente e, apesar de instaladas muito próximas, estas podem apresentar variação quanto às características do solo, uma vez que foram mantidas as condições originais do perfil do solo, sendo preparada somente a camada superficial, em torno de 10 a $20 \mathrm{~cm}$ de profundidade. Os resultados apresentados estão em concordância com os encontrados por Frauenfeld \& Truman (2004), os quais observaram que o perfil de precipitação pluvial não influencia o valor da taxa de infiltração estável.

A tendência de estabilização rápida dos valores da taxa de infiltração na segunda e terceira aplicações pode também ser explicada pelo fato de que, quando o solo está úmido, os agregados não sofrem a quebra devido ao rápido umedecimento e expulsão do ar dos poros interagregados, além de que a água empoçada na superfície do solo protege os agregados do impacto direto das gotas de chuva. Assim, o que causou maior impacto na redução dos valores da taxa de infiltração de água no solo pode ter sido a camada de encrostamento formada na primeira aplicação no solo seco. 
Se comparados os comportamentos das curvas da taxa de infiltração dos ensaios realizados em solo com e sem cobertura vegetal, observa-se que nos ensaios sem cobertura a taxa de infiltração é muito menor, principalmente na segunda e terceira aplicações. Nos ensaios sem cobertura, com as sucessivas aplicações, houve decréscimo muito rápido e acentuado da taxa de infiltração com o tempo; nos ensaios com cobertura, quando houve decréscimo da taxa de infiltração com o tempo, ele foi menos abrupto, e na segunda aplicação praticamente toda lâmina precipitada infiltrou no perfil do solo, sendo os resultados bem contrastantes se comparados aos encontrados em solo nu.

Esses resultados devem-se aos restos culturais sobre a superfície do solo, que, de acordo com Barcelos et al. (1999), interceptam e dissipam a energia cinética da chuva e do escoamento superficial, diminuindo a desagregação e o transporte de partículas. Dessa forma, previnem a formação do encrostamento superficial e diminuem a velocidade do escoamento, aumentando o tempo de contato da água com o solo, proporcionando, portanto, maior infiltração de água no solo.

Deve-se ressaltar o fato de que os conteúdos de água no solo no início dos ensaios eram muito próximos para as parcelas com solo sem e com cobertura e, desta forma, a queda na capacidade de infiltração de água no solo deve-se à formação de encrostamento superficial em solo sem cobertura, e não a diferenças no gradiente do potencial total da água no solo entre as duas condições da superfície do solo.
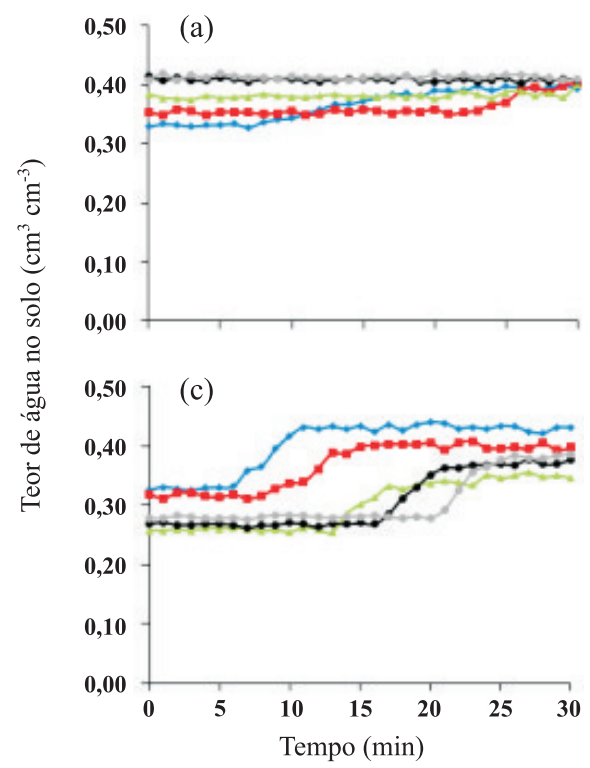

$\because 10 \mathrm{~cm} \rightarrow-20 \mathrm{~cm}$
Comparados os valores da taxa de infiltração estável (Tie) obtida nos ensaios em solo sem cobertura, de $15 \mathrm{~mm} \mathrm{~h}^{-1}$ em média, com a Tie em solo com cobertura, que é de $60 \mathrm{~mm} \mathrm{~h}^{-1}$, nota-se que os valores são bem menores nos ensaios realizados em solo sem cobertura, reduzindo-se a Tie a $25 \%$ do valor obtido em solo com cobertura, sendo aplicada uma energia cinética acumulada da chuva média de $460 \mathrm{~J} \mathrm{~m}^{-2}$.

Jennings et al. (1988), Silva \& Kato (1997) e Barcelos et al. (1999) encontraram resultados semelhantes aos obtidos neste trabalho; segundo Silva et al. (2006), esse comportamento ocorre pelo fato de a condição superficial do solo ser um fator limitante para a infiltração, principalmente quando a superfície do solo encontra-se descoberta, propícia à formação de encrostamento superficial. No trabalho de Silva \& Kato (1997), realizado em um Latossolo argiloso sem cobertura, com a aplicação de $99 \mathrm{~J} \mathrm{~m}^{-2}$ de energia cinética acumulada da chuva, a condutividade hidráulica reduziu-se a $17 \%$ do valor original.

Outro fator que leva à dedução de que houve formação de encrostamento superficial é que, para os ensaios em solo descoberto, para a segunda e terceira aplicações, as quais apresentaram queda abrupta na taxa de infiltração logo no início dos ensaios, a frente de umedecimento alcançou no máximo $20 \mathrm{~cm}$ de profundidade (Figura 5a,b), ao passo que nos ensaios em solo com cobertura, para a segunda e terceira aplicações, a frente de umedecimento alcançou em torno dos $50 \mathrm{~cm}$ de profundidade (Figura $5 \mathrm{c}, \mathrm{d}$ ).

Mesmo com a frente de umedecimento alcançando maiores profundidades, o solo com cobertura
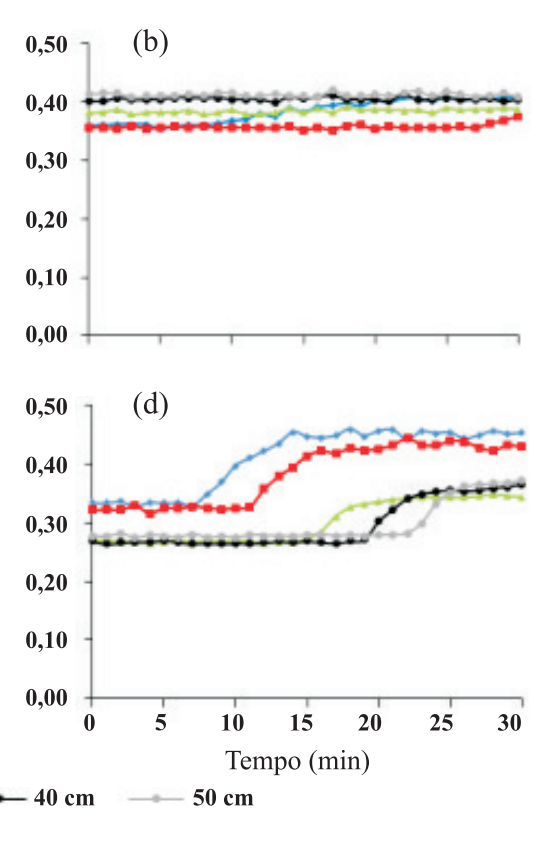

Figura 5. Variação no teor de água no solo com a profundidade e no tempo durante os ensaios de infiltração com perfil de precipitação pluvial constante em solo sem cobertura $-2^{\mathrm{a}}$ (a) e $3^{\mathrm{a}}$ aplicações (b) - e em solo com cobertura - $2^{a}$ (c) e $3^{a}$ (d) aplicações. 
apresentava elevada capacidade de infiltração, demonstrando que essa queda abrupta na taxa de infiltração em solo sem cobertura deveu-se a condições impostas pela camada superficial do solo, como o encrostamento superficial, e não à diminuição do gradiente do potencial total da água no solo.

A fim de avaliar o efeito dos perfis de precipitação pluvial na formação de encrostamento superficial e na infiltração de água no solo, foi analisada a relação entre a lâmina infiltrada e a lâmina aplicada para cada ensaio (Quadro 3).

De acordo com os resultados apresentados no quadro 3, a relação entre a lâmina infiltrada (I) e a lâmina aplicada (L) decaiu da primeira para a terceira aplicação, independentemente do perfil de precipitação pluvial aplicado; com base no teste de média, não houve diferença significativa a $5 \%$ entre os perfis de precipitação pluvial, havendo diferença somente entre as aplicações sucessivas; e os valores para a segunda e terceira aplicações não apresentaram diferenças significativas entre si, diferindo daqueles da primeira aplicação.

Os resultados corroboram os de Parsons \& Stone (2006), Flanagan et al. (1987) e Frauenfeld \& Truman (2004), que não observaram diferenças significativas no valor do volume infiltrado ou escoado comparando diferentes perfis de precipitação pluvial. Segundo Parsons \& Stone (2006), esses resultados ocorrem porque é a energia cinética acumulada da chuva que controla a formação de encrostamento superficial, e não a intensidade de precipitação pluvial. Dessa forma, uma vez que é aplicada a mesma intensidade média e lâmina total, independentemente do perfil de precipitação pluvial aplicado, os efeitos, por exemplo, na formação de encrostamento superficial devem apresentar um mesmo comportamento médio.

Com isso, pode-se afirmar que em solo sem cobertura, exposto ao impacto direto das gotas de chuva, a formação de encrostamento superficial está relacionada com a energia cinética acumulada das gotas de chuva, que está relacionada com a lâmina aplicada. Observa-se também que a formação do encrostamento superficial é mais acentuada nos primeiros minutos ou já na primeira aplicação, visto que para a segunda e terceira aplicações não houve diferença significativa entre os valores da relação entre a lâmina infiltrada e a lâmina aplicada (I/L) (Quadro 3).

Vários autores, como Silva \& Kato (1997), Amorim et al. (2001) e Silva et al. (2001), verificaram experimentalmente que o aumento da energia cinética resulta em redução progressiva da condutividade hidráulica do solo. Brandão et al. (2006b), estudando diversas classes de solos, concluíram que foi evidenciado o desenvolvimento de encrostamento superficial quando aplicados valores crescentes de energia cinética da chuva, caracterizado pela redução da taxa de infiltração, ou seja, a formação de encrostamento superficial foi intimamente ligada à lâmina aplicada.

Isso demonstra que experimentos de perda de água e infiltração, cujo atributo de interesse seja o volume infiltrado ou escoado, podem ser realizados com uso de intensidade de precipitação pluvial constante ao longo do tempo, não acarretando erros nos valores obtidos, mesmo sabendo que as chuvas naturais apresentam intensidade de precipitação pluvial que varia ao longo do evento.

No quadro 4 é apresentada a análise de variância do modelo que descreve o fator de decaimento da taxa de infiltração estável em função da energia cinética acumulada da chuva $(f)$, sendo seus parâmetros de ajuste apresentados no quadro 5 .

De acordo com o quadro 4 , nota-se que a equação apresentou bom ajuste, sendo significativa a $1 \%$ pelo teste F. Com relação aos parâmetros de ajuste da equação, eles foram significativos a $1 \%$ pelo teste t (Quadro 5); assim, a equação ajustada pode ser utilizada para descrever o decaimento nos valores da taxa de infiltração estável em função da energia cinética acumulada da chuva.

Quadro 3. Valores médios da relação entre a lâmina infiltrada e a lâmina aplicada (I/L) para as três aplicações dos diferentes perfis de precipitação pluvial em solo sem cobertura

\begin{tabular}{|c|c|c|c|}
\hline \multirow{2}{*}{$\begin{array}{l}\text { Perfil de precipitação } \\
\text { pluvial }\end{array}$} & \multicolumn{3}{|c|}{ Relação entre a lâmina infiltrada (I) e a lâmina aplicada (L) } \\
\hline & $1^{\mathrm{a}}$ aplicação & $2^{a}$ aplicação & $3^{\mathrm{a}}$ aplicação \\
\hline Constante & $0,97 \mathrm{a}$ & $0,48 b$ & $0,39 \mathrm{~b}$ \\
\hline Exponencial & $0,88 \mathrm{a}$ & $0,34 \mathrm{~b}$ & $0,29 b$ \\
\hline Adiantado & $0,95 \mathrm{a}$ & $0,34 \mathrm{~b}$ & $0,28 b$ \\
\hline Atrasado & $0,87 \mathrm{a}$ & $0,26 \mathrm{~b}$ & $0,25 b$ \\
\hline Média & $0,91 \mathrm{~A}$ & $0,37 \mathrm{~B}$ & $0,3 \mathrm{~B}$ \\
\hline
\end{tabular}


Quadro 4. Resumo da análise de variância para o modelo que descreve o fator de decaimento da taxa de infiltração estável em função da energia cinética acumulada da chuva (f)

\begin{tabular}{lrlrl}
\hline Fonte de variação & GL & QM & F & P \\
\hline Regressão & 2 & 2,31 & 898,56 & $<0,0001$ \\
Resíduo & 33 & 0,0026 & & \\
Total & 35 & 0,1349 & & \\
\hline
\end{tabular}

Quadro 5. Parâmetros da equação ajustada e coeficiente de determinação $\left(R^{2}\right)$

\begin{tabular}{cccccc}
\hline Equação & \multicolumn{2}{c}{ Parâmetro } & $\mathbf{C}$ & $\mathbf{R}^{2}$ \\
\cline { 2 - 3 } & $\boldsymbol{\alpha}$ & $\boldsymbol{\beta}$ & & \\
\hline Exponencial & $0,842^{* *}$ & $0,0061^{* *}$ & $0,157^{* *}$ & 0,989
\end{tabular}

**: Significativo a $1 \%$ pelo teste $t$.

Na figura 6 é apresentado o gráfico relacionando $f$ em função de Ec, com os valores experimentais e ajustados pela equação 3 .

De acordo com a curva gerada, verifica-se que a formação do encrostamento superficial pode ser subdividida em dois estádios: o primeiro, que corresponde à queda brusca de $f$, mais acentuado e abrupto; e o segundo, no qual a curva apresenta comportamento mais suave, com tendência assintótica.

Se comparados os resultados obtidos com aqueles encontrados por Jennings et al. (1988) e Silva \& Kato (1997), que avaliaram o efeito do encrostamento superficial na condutividade hidráulica do solo saturado, verifica-se, em ambos os trabalhos, que a formação do encrostamento superficial é semelhante, isto é, mais intensa nos primeiros minutos da chuva e mais lenta com o passar do tempo. Ainda, se comparado ao trabalho de Silva \& Kato (1997),

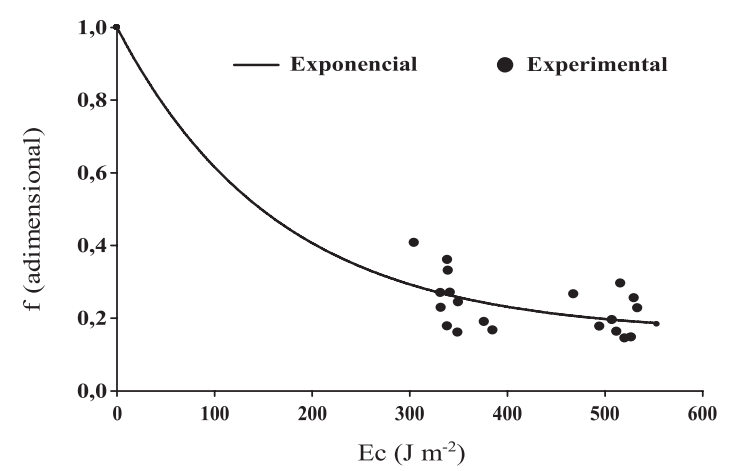

Figura 6. Valores do decaimento da Tie $(f)$ em função da energia cinética acumulada da chuva (Ec). os autores também encontraram decaimento da condutividade hidráulica do solo saturado em função da energia cinética acumulada da chuva, seguindo um comportamento exponencial, corroborando os resultados do presente trabalho.

\section{CONCLUSÕES}

1. A taxa de infiltração de água no solo é influenciada pela lâmina total aplicada e, consequentemente, pela energia cinética acumulada da chuva.

2. A taxa de infiltração estável foi reduzida em $75 \%$ para condições de solo sem cobertura, se comparado ao solo com cobertura.

3. O decréscimo na taxa de infiltração de água no solo devido ao encrostamento superficial pode ser descrito por meio de uma equação do tipo exponencial, sendo função da energia cinética acumulada da chuva.

\section{AGRADECIMENTOS}

À Fundação de Amparo à Pesquisa do Estado de Minas Gerais (FAPEMIG), pelo apoio e financiamento do projeto de pesquisa.

\section{LITERATURA CITADA}

AMORIM, R.S.S.; SILVA, D.D.; PRUSKI, F.F. \& MATOS, A.T. Influência da declividade do solo e da energia cinética de chuvas simuladas no processo de erosão entre sulcos. R. Bras. Eng. Agríc. Amb., 5:124-130, 2001.

ARAÚJO FILHO, J.C. \& RIBEIRO, M.R. Infiltração de água em Cambissolos do Baixo Irecê (Ba). R. Bras. Ci. Solo, 20:263-370, 1996.

BARCELOS, A.A.; CASSOL, E.A. \& DENARDIN, J.E. Infiltração de água em um Latossolo Vermelho-Escuro sob condições de chuva intensa em diferentes sistemas de manejo. R. Bras. Ci. Solo, 23:35-43, 1999. 
BRANDÃO, V.S.; CECILIO, R.A.; PRUSKI, F.F. \& SILVA, D.D. Infiltração da água no solo. 3.ed. Viçosa, MG, Universidade Federal de Viçosa, 120p. 2006a.

BRANDÃO, V.S.; SILVA, D.D.; RUIZ, H.A.; PRUSKI, F.F.; SCHAEFER, C.E.G.R.; MARTINEZ, M.A. \& MENEZES S.J.M.C. Resistência hidráulica da crosta formada em solos submetidos a chuvas simuladas. R. Bras. Ci. Solo, 30:13-21, 2006b.

CUNHA, J.L.X.L. Velocidade de infiltração da água em um Latossolo Amarelo submetido ao sistema de manejo plantio direto. Caatinga, 22:199-205, 2009.

DE ROO, A.P.J. \& RIEZEBOS, H.T. Infiltration experiments on loess soils and their implications for modelling surface runoff and soil erosion. Catena, 19:221-239, 1992.

EMBRAPA MILHO E SORGO - EMBRAPA. Sistemas de produção. 2.ed. v.1. 2006. <Disponível:http://sistemasdeproducao.cnptia.embrapa.br/FontesHTML/Milho/ CultivodoMilho_2ed/mandireto.htm>. Acesso em: 3 de jul. de 2006.

FLANAGAN, D.C.; FOSTER, G.R. \& MOLDENHAUER, W.C. Storm pattern effect on infiltration, runoff, and erosion. Trans. ASAE, 31:414-420, 1987.

FRAUENFELD, B. \& TRUMAN, C. Variable rainfall intensity effects on runoff and interrill erosion from two coastal plain ultisols in Georgia. Soil Sci., 169:143-154, 2004.

JENNINGS, G.O.; JARRET, A.R. \& HOOVER, J.R. Evaluating the effect of puddling on infiltration using the Green Ampt equation. Trans. ASAE, 31:761-768, 1988.

LE BISSONNAIS, Y.; CERDAN, O.; LECOMTE, V.; BENKHADRA, H.; SOUCHERE, V. \& MARTIN, P. Variability of soil surface characteristics influencing runoff and interrill erosion. Catena, 62:111-124, 2005.

MAGUNDA, M.K.; LARSON, W.E.; LINDEN, D.R. \& NATER, E.A. Changes in microrelief and their effects on infiltration and erosion during simulated rainfall. Soil Technol., 10:57-67, 1997.

MEYER, L.D. \& HARMON, W.C. Multiple intensity rainfall simulator for erosion research on row sideslopes. Trans. ASAE, 22:100-103, 1979

MONTEBELLER, C.A. Influência dos perfis de precipitação pluvial nas perdas de solo e água. Viçosa, MG, Universidade Federal de Viçosa, 2009. 63p. (Tese de Doutorado)

MORIN, J.; KAREN, R.; BENJAMINI, Y.; BEN-HUR, M. \& SHAINBERG, I. Water infiltration as affected by soil crust and moisture profile. Soil Sci.,148:53-59, 1989.

PARSONS, A.J. \& STONE, P.M. Effects of intra-storm variations in rainfall intensity on interrill runoff and erosion. Catena, 67:68-78, 2006.

POTT, C.A. \& DE MARIA, I.C. Comparação de métodos de campo para determinação da velocidade de infiltração básica. $R$. Bras. Ci. Solo, 27:19-27, 2003.
PRUSKI, F.F.; VENDRAME, V.; OLIVEIRA, E.F.; BALBINO, L.C.; FERREIRA, P.A.; WERLANG, L. \& CARVALHO, L.T. Infiltração de água num Latossolo Roxo. Pesq. Agropec. Bras., 32:77-84, 1997.

RISSE, L.M.; NEARING, M.A. \& ZHANG, X.C. Variability in Green-Ampt effective hydraulic conductivity under fallow conditions. J. Hydrol., 169:1-24, 1995.

ROBINSON, D.A. \& WOODUN, J.K. An experimental study of crust development on chalk downland soils and their impact on runoff and erosion. Eur. J. Soil Sci., 59:784-798, 2008.

SANTOS, M.R.; ZONTA, J.H. \& MARTINEZ, M.A. Influência do tipo de amostragem na constante dielétrica do solo e na calibração de sondas de TDR. R. Bras. Ci Solo, 34:299-307, 2010.

SILVA, C.L. \& KATO, E. Efeito do selamento superficial na condutividade hidráulica saturada da superfície de um solo sob cerrado. Pesq. Agropec. Bras., 32:213-220, 1997.

SILVA, D.D.; PAIVA, K.W.N.; PRUSKI, F.F.; SCHAEFER, C.E.G.R. \& AMORIM, R.S.S. Escoamento superficial para diferentes intensidades de chuva e porcentagens de cobertura num Podzólico Vermelho-Amarelo com preparo e cultivo em contornos. Eng. Agríc., 21:12-22, 2001.

SILVA, J.C.A.; ANDRADE, A.P. \& SILVA, I.F. Avaliação da infiltração da água no solo como indicador de modificações edáficas em três sistemas de manejo. Agropec. Téc., 27:8591, 2006.

SILVA, L.L. Fitting infiltration equations to centre-pivot irrigation data in a Mediterranean soil. Agric. Water Manag., 94:83-92, 2007

SIMÕES, W.L.; FIGUEIRÊDO, V.B. \& SILVA, E.L. Uso do cilindro infiltrômetro único em diferentes solos. Eng. Agríc., 25:359-366, 2005.

TRUMAN, C.C.; STRICKLAND, T.C.; POTTER, T.L.; FRANKLIN, D.H.; BOSCH, D.D. \& BEDNARZ, C.W. Variable rainfall intensity and tillage effects on runoff, sediment, and carbon losses from a loamy sand under simulated rainfall. J. Environ. Qual., 36:1495-1502, 2007.

WISCHMEIER, W.H. \& SMITH, D.D. Rainfall energy and its relantionship to soil loss. Trans. Am. Geoph. Union, 39:285-291, 1958.

ZONTA, J.H.; MONTEBELLER, C.A.; PRUSKI, F.F.; MARTINEZ, M.A.; SOUSA, H.T. \& FREITAS, M.C. Circuito digital-analógico para automação de um simulador de chuvas. In: CONGRESSO BRASILEIRO DE ENGENHARIA AGRÍCOLA, 36, Bonito, 2007. Anais... Bonito, SBEA, 2007. CD ROM. 
